have developed their knowledge and understanding of practice development methodologies and the impact this can have on developing person-centred cultures.

Aims Use creative practice development methodologies to bring about culture change where current staff engage with possible recruits and feedback into the recruitment process.

To ensure that potential new recruits fully understand the role on which they were embarking and experience these methods in practice during interview.

To improve retention of new staff to this role.

Methods The methods used were based on the Practice Development Workbook (Dewing, McCormack, \& Titchen, 2014). The interview was conducted in three parts; the first two exercises are group activities. Using creativity to understand their journey to here; a values exercise to explore their values as well as Marie Curie's and then a short individual interview. Results It has been challenging at times as the interview process is around three hours rather than the traditional style of interview of at least one hour. Feedback received from individuals who have completed this process have been highly evaluated.

Conclusion To build authentic and meaningful relationships and cultures it is important to identify individual values and beliefs (Wilson, 2011). Establishing person-centred cultures requires a sustained commitment from individuals and organisations. The focus on staff and service users is vital to ensuring compassionate care and a flourishing culture.

\section{P-280 TAPPING INTO THE BENEFITS OF THE ASSISTANT PRACTITIONER}

Hannah Ives. ellenor Gravesend, UK

10.1136/bmjspcare-2017-hospice.305

Background Nationally, the current shortage of nurses is likely to increase, in part due to the cessation of student nurse and other healthcare student bursaries (Imison \& Dayan, 2016). To address this workforce gap, organisations have re-evaluated the role of health care assistants (HCAs) and their potential to develop in the work place (Skills for Health, 2015). Assistant Practitioners work at Band 4, with increased knowledge and skills developed to enable them to assess, analyse and support care needs beyond the traditional role of healthcare assistants (Skills for Health, 2015). Yet little is known if, and how, the Assistant Practitioners would aid the staffing shortfall.

Aims - To identify benefits of Assistant Practitioner role in a hospice setting.

- To successfully embed an Assistant Practitioner within the team.

Methods - A combined approach was used to capture relevant information, including:

- Case studies around Assistant Practitioners

- Current career development pathways and guidelines for Assistant practitioners

- Job descriptions compared

- Questionnaire generated and conducted within a hospice

- Clear job description and pathways created.

Results Benefits of an Assistant Practitioner were widely recognised particularly: greater ability to support and assist care ; significance in increased work responsibilities; staff retention and career progression. Organisations adopted different ways to implement the Assistant Practitioner role demonstrating the diversity possible, including specialising in one area of work. Previous pitfalls centred on lack of communication about the role and responsibilities, acting as a barrier for staff acceptance. Our decision was appoint a new Assistant Practitioner with clear remit and responsibilities. Early benefits we are experiencing include:

- Supporting HCAs specifically with training and competencies

- Freeing specialist nurse time

- Bridging gap between staff grades.

Conclusion Assistant Practitioners can integrate into hospice teams, improving skill mix. Whilst the role continues to evolve, this generates exciting opportunities to develop and adapt work streams; early days but positive signs.

\section{P-281 DEVELOPING THE ROLE OF THE LYMPHOEDEMA ASSISTANT PRACTITIONER AT ST CATHERINE'S HOSPICE}

Ann McFarlane, Debbie Murphy. St Catherine's Hospice, Lancashire, UK

\subsection{6/bmjspcare-2017-hospice.306}

Background St Catherine's Hospice has supported the development of an assistant practitioner within its lymphoedema service as part of a workforce development strategy.

Aims To develop the role of the current support worker to address the skills shortage and recruitment difficulties within the specialty of lymphoedema

To free the specialist nurses to focus on more advanced work.

Methods To ensure the continued provision of high quality patient care, the retention and development of their workforce and taking into account the impact of current financial pressures, a workforce development analysis was done to identify where changes needed to be made. It was identified that the development of the current support worker into an assistant practitioner role was critical. Funding for the project was sought from the apprenticeship scheme and agreement with the senior management team. The education department facilitated the enrolment process with University of Central Lancashire.

Results The evaluation process has not been fully developed at this stage as the training is still in progress.

The following benefits have been identified:

- A clear well defined career pathway

- Specific set core competencies have been developed in line with Skills for Health; this has enhanced multi-disciplinary team working based on clarity of role responsibilities and levels of accountability

- Creating investment in staff training and development increases the recruitment and retention of staff therefore gives job satisfaction

- Individual personal development through increasing knowledge and confidence.

Conclusion The impact on the service has been evident in terms of staff absence for protected learning time as we are such a small team and backfill has proved to be difficult. A reduction in patient treatments as the support worker carries out the low-level interventions. Protected time for clinical learning has also been difficult at times and has been a strain on an already busy department. 


\section{P-282 RENNIE GROVE HOSPICE CARE PRECEPTORSHIP} PROGRAMME

Jo Clarke. Rennie Grove Hospice Care

\subsection{6/bmjspcare-2017-hospice.307}

Background The hospice has developed a preceptorship programme in response to the national shortage of nurses and the increasing demand for its service with increase in referrals year on year. We have begun to see the effects of the national shortage in nurses in our own recruitment. After 30 years of caring for our patients and having asked for experience in palliative care or community experience we decided to make the move away and recruit newly qualified nurses after a robust recruitment process.

Aims - to develop a preceptorship programme for newly qualified staff to run over 12 months

- write a job description and role profile

- write interview questions, marking grid and scenarios for interview

- write a competency framework

- prepare the existing workforce of 90-strong nurses to support and co-mentor the preceptees

- write preceptee guidelines and prepare the preceptors for their new roles

- run an initial pilot with two preceptees.

Method Completed a literature search around preceptorship programmes in the community and hospice arenas; explored what was happening in other areas; looked at the NMC code and aligned competencies with the code; aligned competencies with e-lfh; used the RCN framework for nurses working in specialist palliative care; attended job fairs at the local universities to advertise the programme.

Results The interviewing process and questions was effective in selecting two candidates; the preceptorship programme and frame work has yet to be tested; a full-12 month programme is ready for successful candidates to undertake.

Conclusion We are still looking to recruit suitable candidates for the programme and have identified that we would be in a stronger position if we were to take third year students and those doing the return to practice course. To this end we have approached two universities to support existing mentors to become 'sign off' mentors.

\section{P-283 THE UNIQUE ROLE OF THE NURSE PRECEPTOR IN COMMUNITY PALLIATIVE CARE}

Melanie Legg. Marie Curie, London, UK

10.1136/bmjspcare-2017-hospice.308

Background For patients approaching end of life, the majority have a preference to be cared for and to die at home (de Roo et al., 2014). Community palliative care makes a key contribution towards this goal. With the increased demand on these services, there will be an increase in the number of registered nurses and health care support workers needed to meet these needs. As an organisation we need to support this through initiatives to aid the recruitment, retention, job satisfaction and performance of staff working in this setting. There is a lack of previous research on the impact of transition to palliative community care on the member of staff. As far as the research team is aware, this is the first study to explore the role of the nurse preceptor in palliative care with both registered nurses and health care assistants.

Aims To develop an understanding of the nurse preceptor role in community palliative care to explore the experience of the nurse preceptor in community palliative care to identify the barriers and rewards of undertaking this role.

Method All nurses $(n=12)$ undertaking the preceptor role were approached an invited to participate in the research study. 7 preceptors participated over two scheduled focus group sessions, two withdrew from preceptorship prior to the focus groups. At the end of the focus group interviews the participants were debriefed.

Results Four key themes were identified:

supporting the individual, promoting clinical readiness, confidence building, and advocacy and the preceptor.

Conclusion Preparation of new staff into the role of a community palliative health care worker is essential to development, support and integration of the individual into the new working environment as well as the organisation. Lone working is a requirement within our community palliative care services and was identified as a major concern in focus groups as well as the potential impacts and scope for development in relation to this.

\section{P-284 DIPPING A TOE IN THE WATER: DEVELOPING PROFESSIONAL ROLES FROM WITHIN}

Helen Birch, Karen Groves, Catherine Ainsworth, Laura Webster. Queenscourt Hospice, Southport, UK

\subsection{6/bmjspcare-2017-hospice.309}

Aim Experienced, qualified and unqualified, hospice nurses desire career progression which is limited within the hospice environment. Time and money is invested in education to deliver high quality palliative care. For nurses to then take those skills elsewhere leaves a huge gap in our workforce. An NHS post is the usual route to becoming a Palliative Care Nurse Specialist (PCNS). If experienced Health Care Assistants (HCA) want to progress they are currently required to attend university to gain an academic qualification and for many this would prove challenging.

Approach Local hospices' professional development was scoped, but revealed no specific models and new roles were bespoke. Two local roles were developed; Hospice Nurse Specialist (HNS), and HCA Therapy Assistant (TA). Roles were clearly defined, with robust job descriptions and person specifications, scope of practice agreed, required skills, competences and boundaries established.

Result The skilled HNS provides continuity of care for patients and a visible presence provides a vital link between nursing and medical professionals. It has led to a more streamlined admission process for the patient and following completion of the non-medical prescribing module, the HNS works autonomously wherever the patient presents, allowing medical staff to focus on patients with more specialist complex needs. The TA role presented its own challenges as there is no national specifically defined pathway to follow. Specific competencies were developed including input from community equipment suppliers, wheelchair services and by shadowing various therapy teams within hospital and community setting. Conclusion This has been a successful 'dip of the toe in the water' for role development. Future development includes an 